\title{
EfFicient Cloud Gaming SCHEMe Using SCENE OBJECTS ADAPTATION
}

\author{
Ahmad A. Mazhar \\ Department of Computer Science, Saudi Electronic University, Taif Branch, KSA
}

\begin{abstract}
The last decade witnessed wide-spread of internet and on-line gaming. Improving the broadband limitations is considered one of the most important factors that led to this extent. The recently huge usage of internet applications and games also led to grow up more the usage of handhelds and smart phones as well. This improvement of internet speed with the limitations of the smart end devices led to rely more on processing games away from these limited devices to robust processing centers in a technique so-called Cloud Gaming. The growth of players number and game content request new ideas of bit rate reduction of the streaming video of these on-line games. In this paper, a new technique has been proposed to reduce the requirements needed by scene customization encoding with negligible impact on playing quality..
\end{abstract}

\section{KEYWORDS}

Cloud Gaming, DSIS, Content Adaptation, H.265, Video Encoding, Video Streaming

\section{INTRODUCTION}

The term Cloud Gaming is upcoming to be one of the most important terms in on-line gaming as it implements cloud computing benefits for gaming. The idea behind cloud gaming is by running the game as real-time application on away gaming centers while streaming the needed requirements to the user's limited device $[1,2]$. The user's device are only required to process a specific amount of data that reflects user's reactions to the game. The huge amount of data processing such as 3D graphics rendering, are not necessary to be done on the client's device [3].

The streaming of a game can be done typically by streaming objects and graphics to the player along with updated messages. This approach consumes a lot of bandwidth and needs higher requirements for users' devices. Another way is done by encoding the game as a video stream before it is communicated with users. The third approach depends on streaming game video and the content graphics in hybrid technique. All previous approaches are considered efficient but require high hardware specifications by the end-user [4].

The new trend of on-line gaming and the term of cloud gaming is to minimize dependency on the end-user hardware. The end-user should not tolerate the huge amount of processing on his/her device. A high-speed internet connection is the only thing that the end-user needs to have. Fortunately, the vast broadband improvement makes this requirement available in different places around the world $[5,6,7]$.

According to this technique, end-user needs only to send control inputs of a game to the game server and receives the game video on his own device. The game texture is encrypted and sent to the user as an encrypted video sequence. In this case, the game server on the cloud performs the time consuming processes such as video compression and objects rendering. Thus, high internet speed and ability of high quality video display are the only requirements by the user side. However, new platforms are needed to be designed for these upcoming techniques $[8,9,10]$.

DOI:10.5121/ijcsit.2017.9215 
The new video gaming trend deals with the game as a video sequence not as a game and can be called as a Game as Video technique. The game as video technique provides also more advantages than only less hardware requirements by user. The use of console gaming is not required where users need only to deal with a video sequence with only relatively cheap controllers such as joystick. Users should only have a device to display video with ports for such controller, if needed, and no need for updating consoles or spending money on buying game discs. In this case, user's device can be a smartphone, tablet or any other device with video displaying ability.

Even though the computer gaming has grown up hugely, the number of mobile gamers has increased throughout the forgoing years. That indicates on the increasing of the importance of exploiting this field by game developers. In the current market, there are many cloud gaming services available. Some of these services depend on PCs with a window application such as OnLive. However, some PCs services depends on a web application as in GaiKai [11]. Modern services such as GameNow and PSNow became more competitive in this field of gaming [12, 13, $14,15]$. TV accesses services that are more modern, which use special connectors and controllers to make users able to play by just connecting these TVs to the Internet without needing a game console. However, the most modern and widespread services depend on mobiles with cloud gaming. This is one of the most important and updated services that will have more focus in the future. Based on the wide spread of smart phones in addition to the increased number of mobile gamers, cloud gaming using mobiles and handheld computers became more popular and easy to use by users. Only in the USA, the number of mobile gamers raised from 78 million in 2009 to 181 million in 2016. These numbers are very important indicators on the fast growth of mobile game players and led to consider this growth as the fastest in the field of digital gaming. In the business market, the mobile gaming has been under the focus of game developers and industry observers. According to the ABI research report, the mobile gaming revenues will grow to more than 20 billion USD by the end of 2017. Since both industry and technology indicated that online gaming is an active and potential upcoming field, the mobile gaming will grow more and is getting much popular. As a result, the cloud gaming is expected to become more usable because it decreases the usage of hardware required by user. One more important factor is the decreasing of gaming cost as the user can deal with the game developer directly.

As cloud gaming provides many benefits by reducing hardware required to run a game and increasing revenue for developers, unfortunately, some problems advanced while applying this technique. Bandwidth limitations and transmission latency affected poorly on the quality of the service. This problem occurs as the most parts of rendering are done on the cloud and not on the user's device as in the conventional way. This increases demanding on video game streaming and then the more bandwidth consuming and more network resources needed. As a result, the network limitations will play an important factor on the game quality and enjoyment.

In this paper, a novel algorithm is introduced to control the bit rate streaming. The devised algorithm focuses on adapting the content of a game scene to reduce the amount of data required to be sent by selecting partial contents of the game scene. Reducing the amount of non-effective objects that need to be transmitted will save network bandwidth and processing time.

The paper is organized as follows: section II presented a survey of the previous works in the literature. Section III discusses the proposed algorithm with description. Intensive experiments with result analysis are shown in section IV. Finally the paper is concluded in section V. 
International Journal of Computer Science \& Information Technology (IJCSIT) Vol 9, No 2, April 2017

\section{Previous WORKS}

As a game can be streamed as a video sequence in the game video technique, there is a need to adapt this sequence and to be able to implement it in different environments. In [16], a new methodology to improve player's satisfaction is presented. The proposed mechanism adjusts the game parameters in a real time manner to improve the entertainment for the player. A commercial analysis of NVIDIA GeForce NOW game streaming is conducted in [17]. The analysis highlights the problems of network limitations and proposed an adaption mechanism of game streaming considering the end user network constraints. An empirical study to evaluate playing quality is conducted and concludes that new future video encoding adaption strategies can be recommended. In [18], a variant of Temporal Coherence Learning with geometric step size changing was proposed. They showed its algorithm outperformed other ones with constant change of step size. Some new researches focused on the cloud computing as an important factor on speeding-up on-line video gaming. In [19], the paper minimized the end-to-end distortion of real-time communication of the video and devised a new technique to minimize the loss of rate approximation and source rate control. In [20], a depth analysis of many factors that affect game players have been studied and analyzed. As a result, a high accurate player experience was predicted for both long and short game segments. As the cloud computing is increasingly used in different computing areas, cloud gaming is also becoming an insistent to solve huge number of thin clients who use online gaming. In this circumstance many techniques were proposed and devised to improve cloud gaming. A new request dispatching algorithm was proposed in [21]. The algorithm assigns the required requests after predicting ending times of a game session. The predictions were conducted using neural network algorithm. In [22], authors presented a new technique based on live rendering. The rendering supports inter-frame and intra-frame compression. This technique reduced the demanding on the bandwidth and gained bandwidth saving up to $73 \%$ compared to raw graphics streaming. In [23], a new algorithm to enhance the cloud gaming platform was proposed. The algorithm considered problems in current cloud gaming platforms such as supporting reconfiguration of video codecs dynamically. One important result of the algorithm is maximizing average gamer experience. An extended work of the previous technique called "CloudUnion" was presented in [24]. The work studied in-depth the previous technique and its internal mechanism to solve hidden problems in other systems. An intensive comparison study was conducted with a cloud gaming systems so-called "GamingAnywhere". The work results showed important factors on infrastructure and user behavior that should be taken into consideration when designing a cloud gaming approach. In [25], a hybrid cluster new technique depends on the user-level virtualization was proposed. Firstly, according to resource consumption, they put games in two types: CPU-critical and memory-critical. Secondly, several scheduling strategies were tested and the best scenarios were recommended to be used.

Wireless networks became more popular during last few years. These wireless network technologies have to become implementable for the modern multimedia applications. Video streaming and cloud gaming are the most popular techniques that are being used over the networks nowadays. To update wireless technologies to be able to implement such techniques, many works were proposed. In [26], a new application was presented. The idea focused mainly on error correction over TCP in wireless networks. They analyzed the frame-level distortion and then proposed a new joint distortion estimation solution. The proposed technique showed improvement on the quality of video streaming. In [27], an extended application-layer error correction-coding framework was proposed and improved. Using simulation of real-time gaming, the proposed solution was able to reduce frame rate drops and the random loss of packets.

Many ideas were proposed in the literature on the cloud gaming. However, few ideas were on the idea of game texture adaptation. This work, in the next section, proposes a new idea on adapting the video of a game to reduce resources and bandwidth consumption. 


\section{Proposed Algorithm}

The main idea behind this proposed idea is to render the effective 3D game objects on the cloud instead of render it on the client side. Once the game objects are rendered and encoded on the cloud with higher computer specifications, the clients are required only to receive a standard encoded video stream and decode it in order to display on their screens. Therefore, we devised a new idea to take down the streamed bit rate by embedding a fast encoding algorithm [28] and to reduce encoding time by adapting the game scene. The idea based mainly on reducing the scene contents by eliminating the non-effective objects. This content reduction will decrease both rendering and encoding time. Also, it will minimize the required bit rate needed to be transmitted between cloud and clients. The rendering and encoding techniques are run on the server as a cloud gaming approach. The proposed encoding technique in [28] is recommended to be used for faster encoding process. This is done by capturing a video scene and encode it on the cloud, then transmit it to the client. The excluding of less important contents in a game scene depends mainly on the player's actions and events. Determining of these important actions are recorded and captured by sensing player's actions in each frame then prepare a dynamic list of important scene contents. The auto-generated list of all objects is updated and sorted dynamically to give the highest priority to the objects that are being used more by the player. The most important contents that were noticed by our analysis stage were expected to be important in player decision, such as: avatar, gun, monster in attack and other dynamic objects. Lesser-used objects are given lower priority; the objects may contain background, sky, terrain and other complementary objects in the scene. After determining the content and ordering objects according to its importance, a set of parameters are specified to give criteria on what objects to be included in the rendering process. The criteria based mainly on the specifications of the network capability and the end-user device specification.

The proposed algorithm shows a combination of a fast encoding technique [28] with the proposed object adaptation technique. As the adopted encoding algorithm is already more-rapidly, and it is proposed to be run by the cloud, the encoding time became faster and acceptable for cloud gaming. In addition, the adaption technique gives more flexibility to choose the important objects depending on the resources available. As a result, these considerations will guarantee the optimal player experience depending on the network and devices available. The proposed algorithm proceeds as in the following outline.

\section{Object adaptation algorithm:}

1. Sensing player's actions on the game.

2. Using sensed of actions, build/update a priority list of important objects in the content by sensing objects that have been used higher by user.

3. According to player's network and device capabilities, determine the most important objects to share from cloud to player.

4. Using technique in [28], encode objects that were selected in Step 3.

5. Append encoded objects to the scene and stream to the player.

6. If game is still on, go to Step 1.

The proposed algorithm shows that the priority list is dynamic and updated automatically each a set of frames. This leads to keep sensing the player's actions and guarantees to determine the importance of the objects related to the scene. Moreover, high content scenes will focus on the most important objects that affect the player experience with minimum resources consumption. 


\section{EXPERIMENTS AND ANALYSIS}

The proposed algorithm has been evaluated using Call of Duty game as it is considered one of the most popular game in the world [29]. The proposed algorithm has been implemented to create a priority list of objects in the game scenes. The highest priority objects have been encoded, rendered and appended to the game stream. Some of the scene components were not selected and have been eliminated as they do not affect player decisions. For example, some details of the terrain and sky are not necessary while a player is standing and shooting on target. Figure 1, shows three original and adapted scenes captured from the game during actual playing.

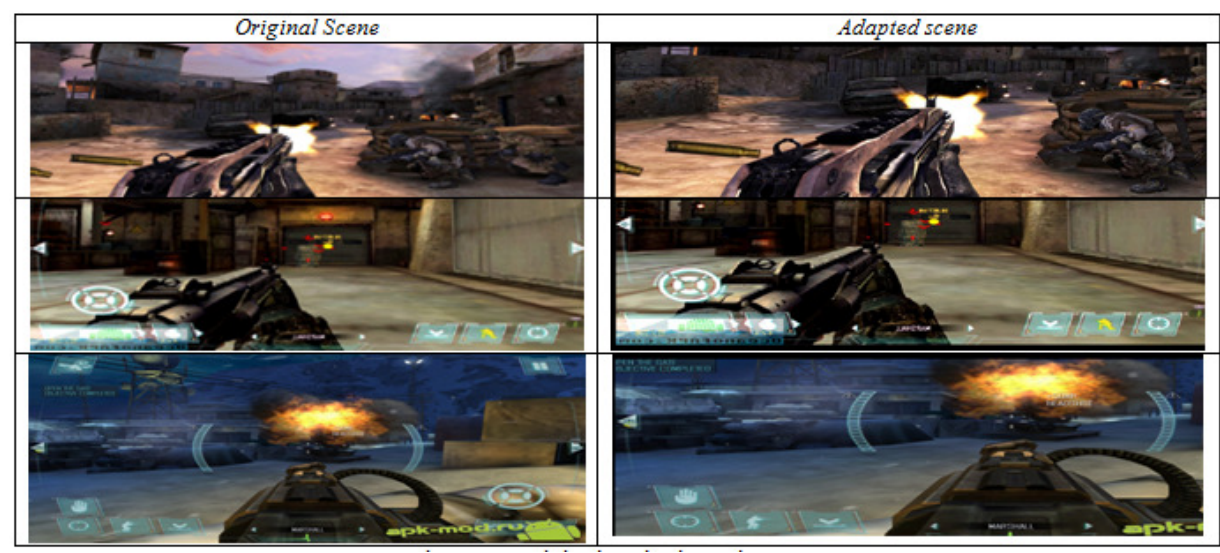

Figure 1. Original and adapted scenes

As seen in figure 1, the irrelevant components that do not affect player decision have been eliminated. The game was played for 60 seconds, the playing scene was being captured using Fraps [30] with $30 \mathrm{fps}$ and $1024 \times 920$ pixels resolutions. The captured sequences were encoded using [28] with the parameters shown in Table 1. The encoding algorithm and parameters were chosen as they give high compression performance with acceptable encoding time.

Table 1 Encoding settings

\begin{tabular}{|l|l|}
\hline Name of the Parameter & Value \\
\hline No. of references & 5 \\
\hline No. of encoded frames & 150 \\
\hline Motion vector search range & 16 \\
\hline Motion vector resolution & $1 / 4$ \\
\hline Quantization parameter & 28,36 \\
\hline RDO & On \\
\hline GOP & IPPP \\
\hline Bit rate & $1 \mathrm{Mbps}$ \\
\hline
\end{tabular}

After the proposed algorithm was run, it showed interesting improvements on both encoding time and streaming bit rate. The gained results were computed and compared to the original video stream. The compares are shown in Table 2. 
Table 2 Comparison between original and adapted videos

\begin{tabular}{|l|l|l|l|}
\hline & $\begin{array}{l}\text { Original } \\
\text { Video }\end{array}$ & Adapted Video & $\begin{array}{l}\text { Percentage of } \\
\text { Decrement }\end{array}$ \\
\hline Average streaming bit rate & $1172.5 \mathrm{Kbps}$ & $844.7 \mathrm{Kbps}$ & $27.9 \%$ \\
\hline $\begin{array}{l}\text { Average Encoding time per } \\
\text { Frame }\end{array}$ & $56.3 \mathrm{~ms}$ & $27.8 \mathrm{~ms}$ & $50.6 \%$ \\
\hline
\end{tabular}

As seen in Table 2, the streaming bit rate of the adapted video is decreased by a rate of $27.9 \%$ compared to the original one. In addition, the average encoding time is decreased from $56.3 \mathrm{~ms}$ in the original video to $27.8 \mathrm{~ms}$ in the adapted video by a rate of $50.6 \%$. Even though the saving in both streaming bit rate and encoding time are significant, these savings are expected to be more effective on the cloud as hundreds or thousands of players are served.

The encoding time is a significant factor while encoding real time games. However, combining fast encoding time with an efficient adaption technique will lead to lower bit rate and then reduced bandwidth requirements. The adapted video with fast encoding technique gives an important contribution in the field of cloud gaming. As any small amount of bit rate reduction or encoding time saving lead to massive saving on the cloud because the huge number of clients.

\section{CONCLUSION}

A fast object adaptation technique for cloud gaming was presented. The idea of selecting the important objects in the gaming scene was exploited to eliminate less important and effective objects. Experimental results showed that the proposed technique succeeds to reduce both bit rate and encoding time by rates of $27.9 \%$ and $50.6 \%$ respectively. The proposed adaptation technique is combined to a previous proposed fast encoding technique to achieve higher bit rate reduction and network bandwidth saving.

\section{REFERENCES}

[1] W. Cai, H. Chan, X.Wang, And V. Leung, "Cognitive Resource Optimization For The Decomposed Cloud Gaming Platform,” Ieee Trans. Circuits Syst. Video Technol., Vol. 25, No. 12, Pp. 2038_2051, Dec. 2015.

[2] D. Mishra, M. El Zarki, A. Erbad, C.-H. Hsu, And N. Venkatasubramanian, "Clouds + Games: A Multifaceted Approach," Ieee Internet Comput., Vol. 18, No. 3, Pp. 20_27, May 2014.

[3] W. Cai, M. Chen, And V. C. M. Leung, "Toward Gaming As A Service," Ieee Internet Comput., Vol. 18, No. 3, Pp. 12_18, May/Jun. 2014.

[4] L. Riungu-Kalliosaari, J. Kasurinen, And K. Smolander, “Cloud Services And Cloud Gaming In Game Development," In Proc. Iadis Game Enter-Tainment Technol. (Get), Prague, Czech Republic, Jul. 2013, Pp. 1_10.

[5] S.-P. Chuah, C. Yuen, And N.-M. Cheung, "Cloud Gaming: A Green Solution To Massive Multiplayer Online Games," Ieee Wireless Commun., Vol. 21, No. 4, Pp. 78_87, Aug. 2014.

[6] S. Dey, "Cloud Mobile Media: Opportunities, Challenges, And Directions," In Proc. Ieee Conf. Comput., Netw. Commun. (Icnc), Maui, Hi, Usa, Feb. 2012, Pp. 929_933.

[7] Z. Wu, "Gaming In The Cloud: One Of The Future Entertainment," In Proc. Interact. Multimedia Conf., Southampton, U.K., Jan. 2014, Pp. 1-6.

[8] K. I. Kim, S. Y. Bae, D. C. Lee, C. S. Cho, H. J. Lee, And K. C. Lee, "Cloud-Based Gaming Service Platform Supporting Multiple Devices," Etri J., Vol. 35, No. 6, Pp. 960_968, Dec. 2013.

[9] C.-Y. Huang, K.-T. Chen, D.-Y. Chen, H.-J. Hsu, And C.-H. Hsu, "Gaminganywhere: The _Rst Open Source Cloud Gaming System," Acm Trans. Multimedia Comput., Commun., Appl., Vol. 10, No. 1s, Pp. 10:1_10:25, Jan. 2014.

[10] C.-Y. Huang, C.-H. Hsu, Y.-C. Chang, And K.-T. Chen, "Gaminganywhere: An Open Cloud Gaming System," In Proc. Acm Multimedia Syst. Conf. (Mmsys), Oslo, Norway, Feb. 2013, Pp. $36 \_47$. 
[11] (Jan. 2017). Gaikai. [Online]. Available: Http://Www.Gaikai.Com/

[12] (Jan. 2017). Onlive. [Online]. Available: Http://Www.Onlive.Com.

[13] (Jan. 2017). Playstation Now. [Online]. Available: Http://Www.Playstation.Com/EnUs/Explore/Playstationnow.

[14] (Jan. 2017). Gamenow. [Online]. Available: Http://Www.Ugamenow.Com.

[15] (Jan. 2017). G-Cluster. [Online]. Available: Http://Www.Gcluster.Com/Eng.

[16] G. N. Yannakakis, J. Hallam, "Real-Time Game Adaptation For Optimizing Player Satisfaction," Ieee Transactions On Computational Intelligence And Ai In Games ( Volume: 1, Issue: 2, June 2009 ). Pages 121-133.

[17] M. Suznjevic, I. Slivar, L. Skorin-Kapov, "Analysis And Qoe Evaluation Of Cloud Gaming Service Adaptation Under Different Network Conditions: The Case Of Nvidia Geforce Now," Quality Of Multimedia Experience (Qomex), 2016 Eighth International Conference On 6-8 June 2016.

[18] S. Bagheri, M. Thill, P. Koch, W. Konen. "Online Adaptable Learning Rates For The Game Connect4," Ieee Transactions On Computational Intelligence And Ai In Games. Volume:8. March 2016.

[19] J. Wu, C. Yuen, N. Cheung, J. Chen, "Streaming Mobile Cloud Gaming Video Over Tcp With Adaptive Source-Fec Coding," Ieee Transactions On Circuits And Systems For Video Technology. Volume 27, 1st January 2017.

[20] N. Shaker, G. Yannakakis, J. Togelius, "Crowdsourcing The Aesthetics Of Platform Games," Ieee Transactions On Computational Intelligence And Ai In Games. Volume 5, Issue 3. September 2013.

[21] Y. Li, X. Tang, W. Cai, "Play Request Dispatching For Efficient Virtual Machine Usage In Cloud Gaming," Ieee Transactions On Circuits And Systems For Video Technology, Volume 25, Issue 12. December 2015.[22] X. Liao, L. Lin, G. Tan, H. Jin, X. Yang, W. Zhang. B. Li, "Liverender: A Cloud Gaming System Based On Compressed Graphics Streaming," Ieee/Acm Transactions On Networking, Volume 24, Issue 4. August 2016.

[23] H. Hong, C. Hsu, T. Tsai, C. Huang, K. Chen, C. Hsu, "Enabling Adaptive Cloud Gaming In An Open-Source Cloud Gaming Platform," Ieee Transactions On Circuits And Systems For Video Technology, Volume 25, Issue 12, December 2015.

[24] Z. Xue, D. Wu, J. He, X. Hei, Y. Liu, "Playing High-End Video Games In The Cloud: A Measurement Study," Ieee Transactions On Circuits And Systems For Video Technology. Volume 25, Issue 12. December 2015.

[25] Y. Zhang, P. Qu, J. Cihang, W. Zheng, "A Cloud Gaming System Based On User-Level Virtualization And Its Resource Scheduling," Ieee Transactions On Parallel And Distributed Systems. Volume 27, Issue 5. May 2016.

[26] J. Wu, C. Yuen, M. Wang, J. Chen, C. W. Chen, "Tcp-Oriented Raptor Coding For High-Frame-Rate Video Transmission Over Wireless Networks,” Ieee Journal On Selected Areas In Communications. Volume 34, Issue 8. August 2016.

[27] J. Wu, C. Yuen, N. Cheung, J. Chen, C. W. Chen, "Streaming Mobile Cloud Gaming Video Over Tcp With Adaptive Source-Fec Coding," Ieee Transactions On Circuits And Systems For Video Technology. Volume 27, Issue 1. January 2017.

[28] A. Mazhar, A. Abdalla, "Joint Reference Frame Inter-Mode Selection For Fast H.264 Video Coding," Signal, Image And Video Processing, April 2016, Volume 10, Issue 4, Pp 617-623.

[29] (Jan. 2017). League Of Legend Game, [Online]. Available: Www.Leagueoflegends.Com.

[30] [Online]. Available: Http://Www.Fraps.Com.

\section{AUTHOR}

Ahmad A. Mazhar was born in Amman, Jordan in 1980. He has been a member of the College of Computing and Informatics at Saudi Electronic University since 2015. He has more than eight years of academic experience. He received his Ph.D. in 2013 from De Montfort University, UK; his Master's degree in computer science from Al- Balqa' Applied University, Salt, Jordan; and his Bachelor's degree in computer science from Al-Zaytoonah University, Amman, Jordan.

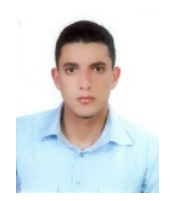

